\title{
Preliminary Discussion on Principle and Application of Thermoelectric Power Generation
}

\author{
Kai Yan \\ North China Electric Power University (Beijing), Beijing 102206, China \\ goodyankaigood@163.com
}

Keywords: Thermoelectric power generation, Seebeck effect, Thermoelectric conversion.

\begin{abstract}
Energy-saving and environmental protection is a serious problem that mankind faced in the 21 st century. In recent years, with the rapid development of economic, energy security situation is increasingly severe. Thermoelectric power generation technology, which is based on Seebeck effect, can directly convert heat energy into electric energy. It is a kind of new and green power generation technology.
\end{abstract}

\section{Introduction}

Thermoelectric power generation technology is a strategic energy and environmental technology in recent years and has been the strong support of most countries. Thermoelectric power generation is mainly applied in the development of high merit looking materials, modules and systems design and optimization aspects.

In the early 1960s, the US Department of Defense space and power systems firm said: 'The thermoelectric power generation is proven to be reliable, low maintenance, long power technology and it can work in extremely harsh environment.' In recent years, some of the successful development of new thermoelectric materials have a higher figure of merit of thermoelectric generator in civilian areas, particularly in the use of energy as a low grade heat source applications, which received wide attention and show its broad application prospects.

\section{Basic principle of thermoelectric power generation}

Thermoelectric power generation uses the Seebeck effect thermoelectric materials, the end of the P-type and $\mathrm{N}$-type thermoelectric materials are connected by an excellent conductive metal $\mathrm{Cu}$; the other end is connected respectively with $\mathrm{Cu}$ conductor constitute as a PN junction, which is also known as PN galvanic arm, as is shown in Fig. 1. In PN galvanic arm, open-side is linked to the load resistance, then flows into the heat. The PN galvanic end of the arm forms a high-temperature side (hot end); the other end of the heat dissipated, the formation of low-temperature side (cold end), so between the PN galvanic arm hot and cold end together establish a temperature difference. Within PN galvanic arm in the high temperature side of the hole (P-type thermoelectric materials) and electronics (N-type thermoelectric material), a temperature gradient was driven, began to low end of the diffusion, thereby PN galvanic arm ends of an electrical potential difference, the circuit there are also current.

EMF is calculated as follows:

$$
\varepsilon=\alpha\left(T_{h}-T_{l}\right)
$$

Where: $\varepsilon$ represents electromotive force; $T_{h}$ represents high-temperature side temperature; $T_{l}$ represents low-temperature side temperature; $\alpha$ is Seebeck coefficient of the thermoelectric material (Seebeck coefficient differs from different materials).

When the temperature difference constant load resistance and the semiconductor resistance almost equally, it reaches the maximum exergy efficiency. When temperature difference increases, exergy efficiency increases. 


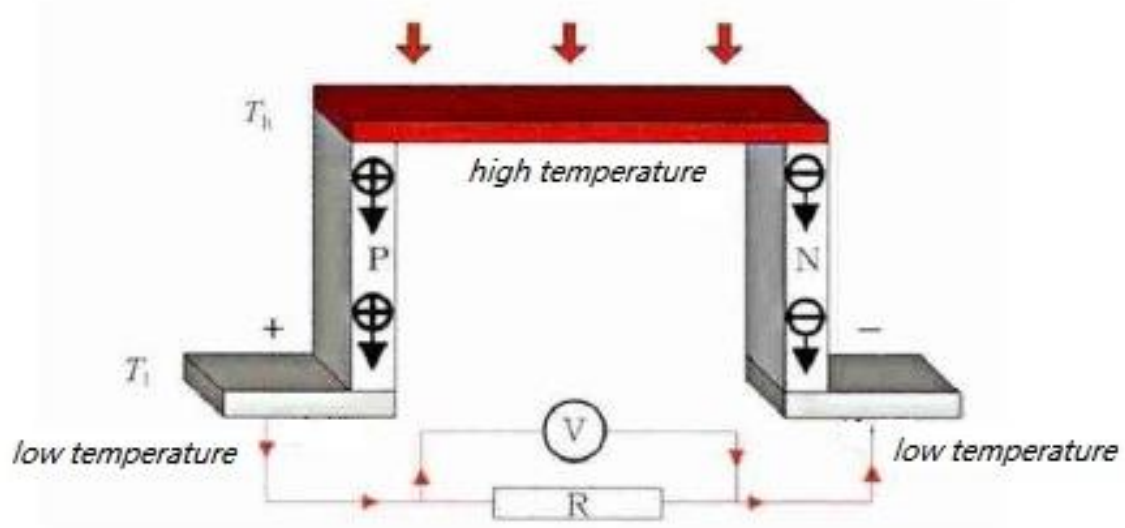

Fig. 1 Principle of thermoelectric power generation

Since a single PN galvanic force arm can produce small electric power, and therefore, in order to obtain a larger output power, a plurality of PN galvanic arm are connected in series to form a thermoelectric module and have a plurality of PN junction. Currently, commercially available thermoelectric component generally looks like a 'sandwich' structure, as is shown in Fig. 2.

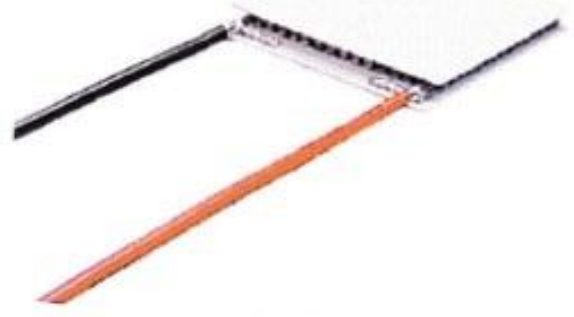

(a) Front view

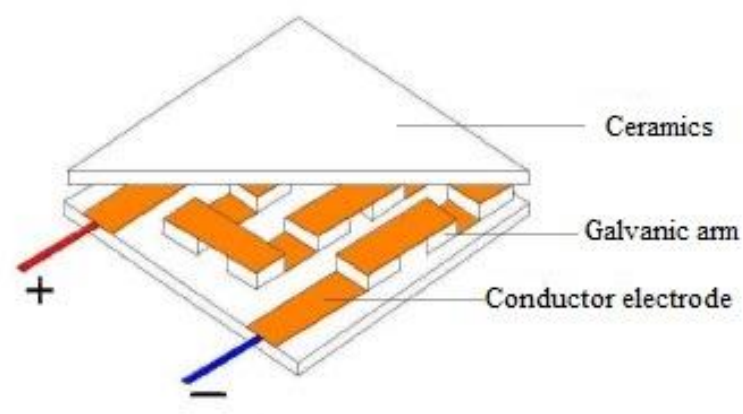

(b) Sectional view

Fig. 2 Schematic diagram of the thermoelectric module

\section{Application of thermoelectric power generation}

\subsection{Semiconductor Thermoelectric power generation}

Semiconductor thermoelectric power generation technology research originated in the $1940 \mathrm{~s}$, first developed by the former Soviet Union successfully. The power generation efficiency is only $1.5 \%$ to $2 \%$. By the 1960s, his research reached a peak, and then semiconductor thermoelectric power generation successfully applied to aerospace and military fields. In the 21 st century, because semiconductor thermoelectric power generation is an environmentally friendly and sustainable form of solid-state thermoelectric conversion technology, civil thermoelectric power generation becomes a very important research direction.

Thermoelectric power generation transferring the Seebeck effect thermal energy directly to electrical energy principle, which is shown in Fig. 3. To improve the power generation efficiency, one method is using a higher Seebeck coefficient of the semiconductor material; another is the introduction of a higher, more stable and constant cold heat source to produce a constant and large temperature difference. 


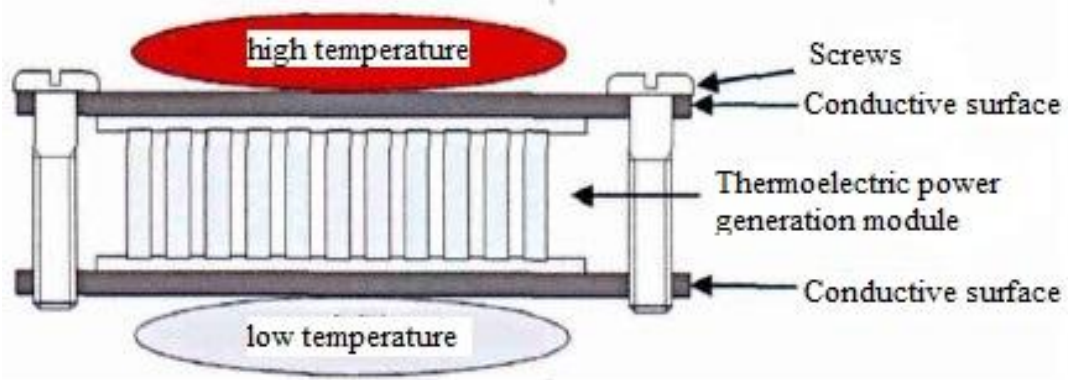

Fig. 3 Semiconductor thermoelectric generator

There is the use of hot water as a heat source semiconductor thermoelectric power generation unit, and use the concentrating solar thermoelectric power generation device as a semiconductor heat source, which is also known as solar collectors thermoelectric power generation unit.

A Thailand scholar in 2004 through the use of a copper plate that was placed on the roof to absorb solar collector heating thermoelectric power generation and the environment, to directly drive axial fans located on the roof, so as to achieve cooling effect to the roof.

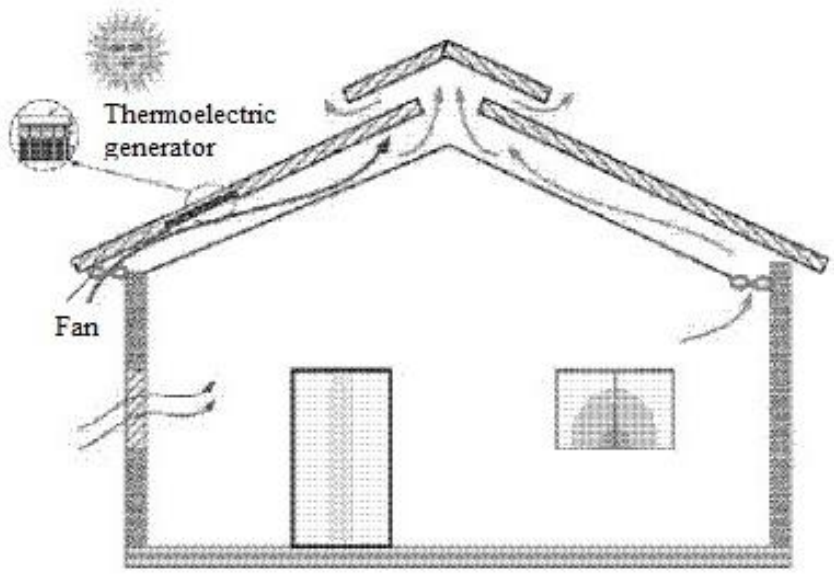

Fig. 4 Schematic diagram of solar thermal power generation roof

\subsection{Ocean Thermal Energy Conversion (OTEC)}

Using ocean surface temperature difference between the water and the cold deep seawater power generation technologies become OTEC. Usually through the atmosphere to reach the sunlight into the sea. About $70 \%$ of the heat is absorbed by seawater below the surface, while the water at the depth of $200 \mathrm{~m}$, barely absorb sunlight and heat. OTEC is the warm ocean surface absorbs nearly $70 \%$ of the sun's heat is evacuated into the inside of the boiler, when the water temperature vacuum boiler immediately vaporized into steam, then take advantage of this warm sea water vaporized into steam driven Turbo power generation, and finally with cold deep sea water after processed, waste steam condensate, is used again. In theory, the temperature difference between the hot water should be higher than $16.6{ }^{\circ} \mathrm{C}$ to generate electricity, and practical applications are generally higher than $20{ }^{\circ} \mathrm{C}$.

\subsection{Other areas}

Thermoelectric power generation technology can utilize solar energy, waste heat successfully and promote their research in other areas, such as geothermal energy, ocean thermal energy, and body heat. In recent years, with the development of microelectronics technology, micro-electromechanical systems (MEMS) is applied to a lot of information and communication, biomedical, consumer electronics, medical and other fields. Small and light, long life and reliable miniature power supply thereby gains a great deal of development. Highly integrated miniature thermoelectric generator has a thermoelectric power density and short response time. Making it as an electric hospital systems or MEMS device has a unique advantage and some of the miniature thermoelectric generators are successively developed. 


\section{Conclusion}

One of the most critical issues is the solution to the temperature difference between thermoelectric power generation. Considering the environment and the use of the Seebeck effect, thermoelectric power generation characteristics, are affected by the number of PN galvanic, the temperature difference between the heat end and the cold end. Compared with the power systems division, thermoelectric power generation has higher resource utilization and lower equipment failure rate. Ocean thermal energy stored is rich, so OTEC has great development value. The advantages in thermoelectric power generation make it to maintain social sustainable development, environmental protection with outstanding results, so new thermoelectric power generation applications in the field of energy will also be invaluable.

\section{References}

[1]. Liqiang Yan, Brief Discussion of Thermoelectric Power Generation [J]. Solar Energy, 2014, p.13-14

[2]. Guangfa Tang, Application and Prospect of Thermoelectric Power Generation Generation [J]. Refrigeration Air Conditioning \& Electric Power Machinery, 2006 112(27), p.9

[3]. Zeguang Zhou, Heat Transfer Characteristic and Mechanism Research of Thermoelectric Generator [D], South China University of Technology, 2013, p.9-10

[4]. Jianyun Zhao, Research and Development of thermoelectric power generation [J], Power Source Technology, 2010 34(3), p.311

[5]. Teng Zhang, Thermoelectric Power Generation Technology and Its Application [J]. Energy Technology, 2009 30(1), p.36 